\title{
ELECTRONIC NEUTRON PERSONAL DOSEMETERS: THEIR PERFORMANCE IN MIXED RADIATION FIELDS IN NUCLEAR POWER PLANTS
}

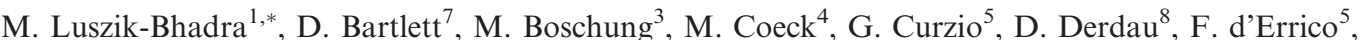

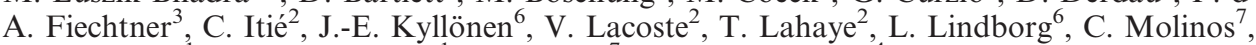 \\ M. Reginatto ${ }^{1}$, H. Schuhmacher ${ }^{1}$, R. Tanner ${ }^{7}$ and F. Vanhavere ${ }^{4}$ \\ ${ }^{1}$ Physikalisch-Technische Bundesanstalt, D-38116 Braunschweig, Germany \\ ${ }^{2}$ Institut de Radioprotection et de Sûreté Nucléaire, F-92265 Fontenay-aux-Roses, France \\ ${ }^{3}$ Paul Scherrer Institut, CH-5232 Villigen, Switzerland \\ ${ }^{4}$ Studiecentrum voor Kernenergie-Centre d'étude nucléaire, B-2400 Mol, Belgium \\ ${ }^{5}$ Dipartimento di Ingegneria Meccanica, Nucleare e della Produzione, I-56126 Pisa, Italy \\ ${ }^{6}$ Swedish Radiation Protection Authority, SE-171-16 Stockholm, Sweden \\ ${ }^{7}$ National Radiological Protection Board, Chilton, Didcot OX11 0RQ, UK \\ ${ }^{8}$ Kernkraftwerk Krümmel GmbH, Elbuferstrasse 82, 21496 Geesthacht, Germany
}

This work describes spectral distributions of neutrons obtained as function of energy and direction at four workplace fields at the Krümmel reactor in Germany. Values of personal dose equivalent $H_{\mathrm{p}}(\mathbf{1 0})$ and effective dose $E$ are determined for different directions of a person's orientation in these fields and readings of personal neutron dosemeters-especially electronic dosemeters-are discussed with respect to $H_{\mathrm{p}}(10)$ and $E$.

\section{INTRODUCTION}

Within the EC project EVIDOS ['Evaluation of Individual Dosimetry in Mixed Neutron and Photon Radiation Fields', see recent overviews ${ }^{(1-3)}$, a series of new electronic neutron personal dosemeters and some passive ones have been irradiated at the nuclear power plant at Krümmel in Germany. Places that are routinely visited for control measurements were chosen: a position in the control rod room underneath the reactor, another position near the top of the reactor and two places near a cask containing used fuel.

Within the project a new spectrometer consisting of six detector capsules - each containing four silicon detectors - mounted onto the surface of a polyethylene sphere was developed ${ }^{(4,5)}$. This allows the determination of the double differential distribution of neutron fluence with respect to energy and direction. Values of ambient dose equivalent and personal dose equivalent $H_{\mathrm{p}}(10)$ for the main direction of incidence have been derived ${ }^{(2)}$ by multiplying the fluence distributions by the corresponding fluenceto-dose conversion coefficients.

This work describes in more detail the directional dose distributions obtained in the different workplaces at Krümmel as well as values for $H_{\mathrm{p}}(10)$ and for the effective dose $E$ for different directions of a person's orientation in the fields. Finally, personal dosemeter readings obtained in these

${ }^{*}$ Corresponding author: marlies.luszik-bhadra@ptb.de workplace fields are presented with respect to $H_{\mathrm{p}}(10)$ and $E$.

\section{THE DIRECTIONAL SPECTROMETER}

The directional spectrometer with silicon detectors is described in detail in Refs (4) and (5). It consists of six detector capsules - each containing a stack of four silicon detectors - mounted onto the surface of a $30 \mathrm{~cm}$ diameter polyethylene sphere and electronics to amplify and record the pulse height spectra of all detectors. The response function of this device has been determined for a series of directions using measurements in quasi-mono-energetic neutron fields and MCNP calculations for neutrons in the energy range from thermal up to $15 \mathrm{MeV}$, and using measurements for photons in the energy region from $80 \mathrm{keV}$ to $6 \mathrm{MeV}$. The pulse height spectra measured in workplace fields are analysed using unfolding codes with respect to energy and direction both for neutrons and photons.

One of the codes (MIEKE) does not take into account pre-information on energy and directional distribution. One unfolding was performed taking the full pulse height information and a combined unfolding for neutrons and photons, a second unfolding was performed taking pulse height signals above a higher threshold and doing an unfolding with respect to neutrons only.

The other code (MAXED) takes pre-information with respect to the neutron energy into account. One unfolding was performed using the spectral shape measured at the same places by Bonner spheres 
(BSs) as pre-information, a second unfolding was performed using other measurements in similar fields as pre-information.

The performance of the directional spectrometer has been checked ${ }^{(4)}$ by measurements in the simulated workplace field CANEL at Cadarache. $H^{*}(10)$ contributions per angular interval, derived from MCNP calculations, decrease sharply above $30^{\circ}$, whereas the results of the directional spectrometer show significant contributions up to $60^{\circ}$. This shows the coarser angular resolution of the experimental device. The fluence $\phi$ and the ambient dose equivalent $H^{*}(10)$ obtained by integrating the contributions per angular interval obtained by the directional spectrometer have been compared with reference values obtained by BS spectrometry. In the case of the MAXED unfolding with BS pre-information, deviations were $<20 \%$.

For the calculation of personal dose equivalent $H_{\mathrm{p}}(10)$, fluences obtained in 14 directions (MIEKE) and in 20 directions (MAXED) were multiplied by fluence-to-dose conversion factors taken from ICRP74 ${ }^{(6)}$ and new calculations for backward incidence $^{(7)}$. Appropriate values for the required energies and angles were obtained by interpolation.

For the calculations of effective dose $E$, the fluences were multiplied by the conversion factors for AP, PA, LLAT and RLAT as given in ICRP74 ${ }^{(6)}$. Values for the directions 'Up' and 'Down' are estimated from the difference between the conversion factors given for ISO and ROT by (3·ISO $-2 \cdot$ ROT). In addition, the influence of revised $w_{R}$ values that are likely to be included in the 2005 recommendations of the ICRP as recently proposed ${ }^{(8)}$ on values of effective dose are estimated. The fluence-to-dose conversion factors given in ICRP 74 for AP, PA, LLAT, RLAT, ISO and ROT have been divided by the $w_{\mathrm{R}}$ values as given in ICRP 74 and multiplied by the newly recommended values.

\section{THE WORKPLACE FIELDS AT KRÜMMEL}

The measurements were performed at two positions near an NTL11 cask with spent fuel and at two positions inside the boiling water reactor (Figure 1).

In Table 1 details on the positions are given, together with reference values for $\dot{H}^{*}(10)$ obtained by BS spectrometry. The last column in Table 1 contains the ratio of neutron and photon contributions of ambient dose equivalent (the latter measured by a FHT $191 \mathrm{~N}$ ionisation chamber). At the cask, neutrons contributed with $>90 \%$ to the ambient dose equivalent. At the position 'KKK TOP' the neutron dose was also dominant, while at the position 'KKK SAR' the photon dose dominated because of the heavy biological shielding.
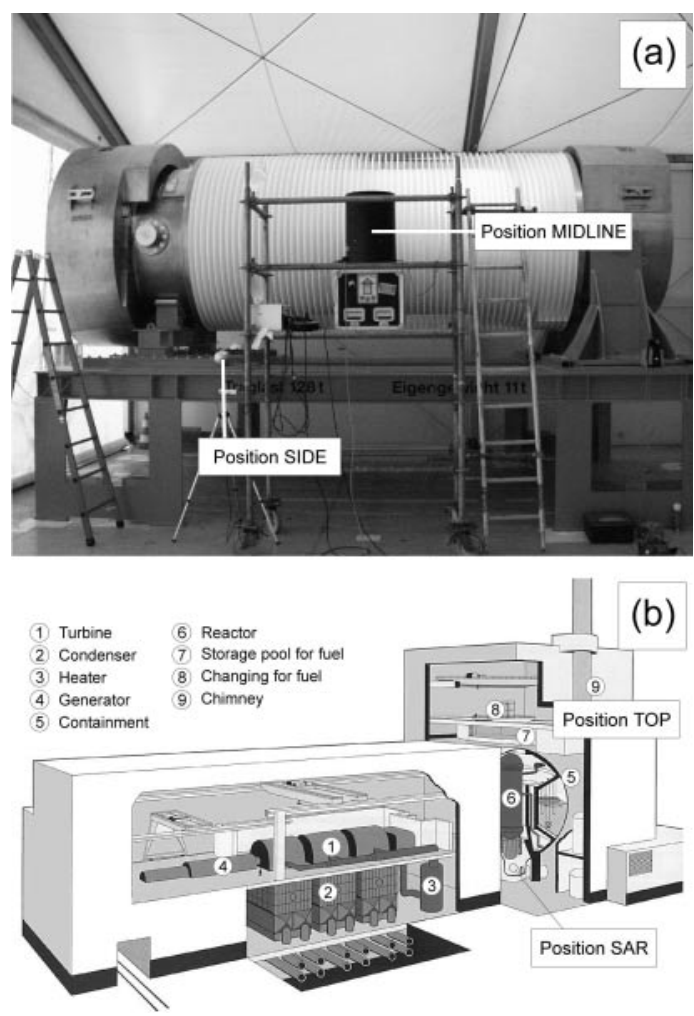

Figure 1. Measuring positions at an NTL11 cask (a) and inside the reactor (b) at Krümmel.

The fluence spectra obtained by BS spectrometry are shown in Figure 2. The spectra at the cask were quite hard, with a main fluence contribution at a few hundred $\mathrm{keV}$, whereas the spectra at the reactor contained a considerable amount of thermal and intermediate energy neutrons. A comparison with fluences obtained by the directional spectrometer (unfolding MAXED) by integrating the contributions per angular interval gave deviations of up to $30 \%$.

\section{RESULTS OF DIRECTIONAL DISTRIBUTIONS OF DOSE}

\section{Relative contributions to $H^{*}(10)$}

Figure 3 shows the relative ambient dose equivalent per angular interval for 14 directions, obtained by the unfolding codes MIEKE and MAXED. The 'Front' direction was towards the cask or the reactor, in case of 'KKK SAR' towards the lock. Six of the directions refer to Front (F), Back (B), Left (L), Right (R), Up (U) and Down (D). The eight directions in between are named by a combination of the abbreviations. 
M. LUSZIK-BHADRA ET AL.

Table 1. Measuring positions at Krümmel.

\begin{tabular}{lllcrr}
\hline Position & Distance 1 & Distance 2 & $\begin{array}{c}\text { Height above } \\
\text { floor }(\mathrm{m})\end{array}$ & $\dot{H}^{*}(10) \mu \mathrm{Sv} \mathrm{h} \mathrm{h}^{-1}$ & $\dot{H}_{\mathrm{n}}^{*}(10) / \dot{H}_{\gamma}^{*}(10)$ \\
\hline Cask midline & Centre of cask & To cask: $1.0 \mathrm{~m}$ & 2.65 & $152 \pm 23$ & \\
Cask side & 10th ring, left side & To cask: 1.35 m & 1.50 & $54.6 \pm 8.2$ & 40 \\
KKK TOP, 40 m level & To door: 0.8 m & To wall: 0.68 m & 1.50 & $39.0 \pm 5.8$ & 4.20 \\
KKK SAR & Centre of room & Centre of room & 1.20 & $46.8 \pm 7.0$ & 0.24 \\
\hline
\end{tabular}

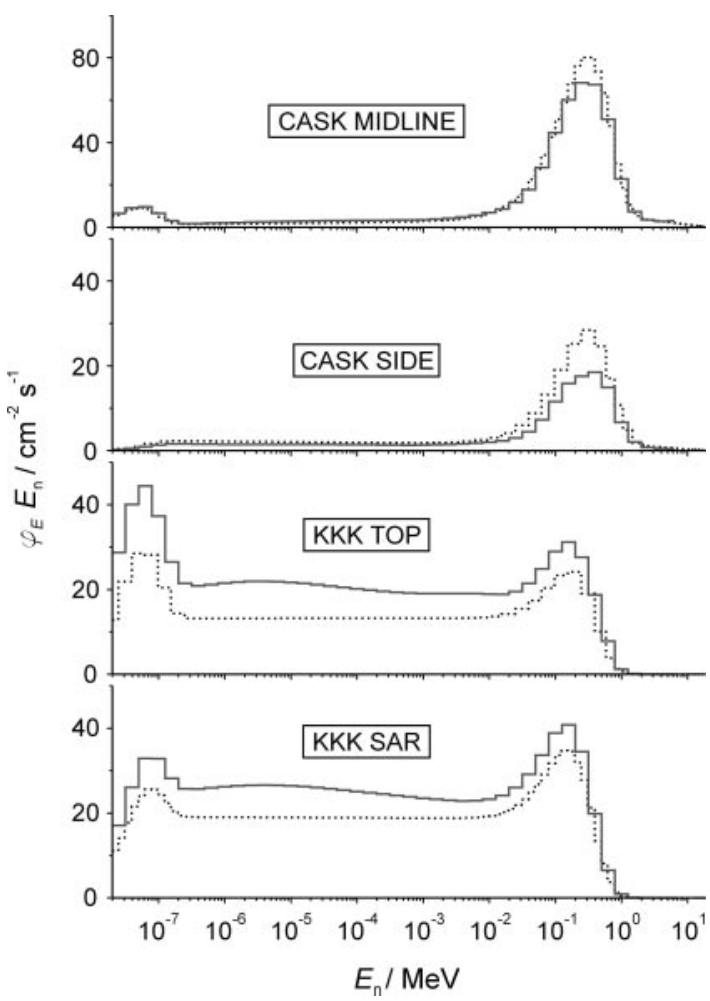

Figure 2. Spectral neutron fluence per logarithmic binwidth as a function of energy at workplaces investigated at Krümmel using BSs (dotted line) ${ }^{(1,2)}$ and the directional spectrometer with silicon detectors (full line, MAXED unfolding with pre-information taken from BSs).

In the case of the position 'cask midline' the distribution decreases by about two orders of magnitude from 'Front' to 'Back'. In the case of the position 'cask side' the decrease from 'Front' to 'Back' is smaller, caused by additional backscatter from the floor. In addition, higher contributions from 'Right' are observed, which is obviously caused by the positioning (Figure 1). In the case of 'KKK TOP' a slightly higher contribution from 'Down' is observed. In the case of the position 'KKK SAR' an almost isotropic distribution is obtained.

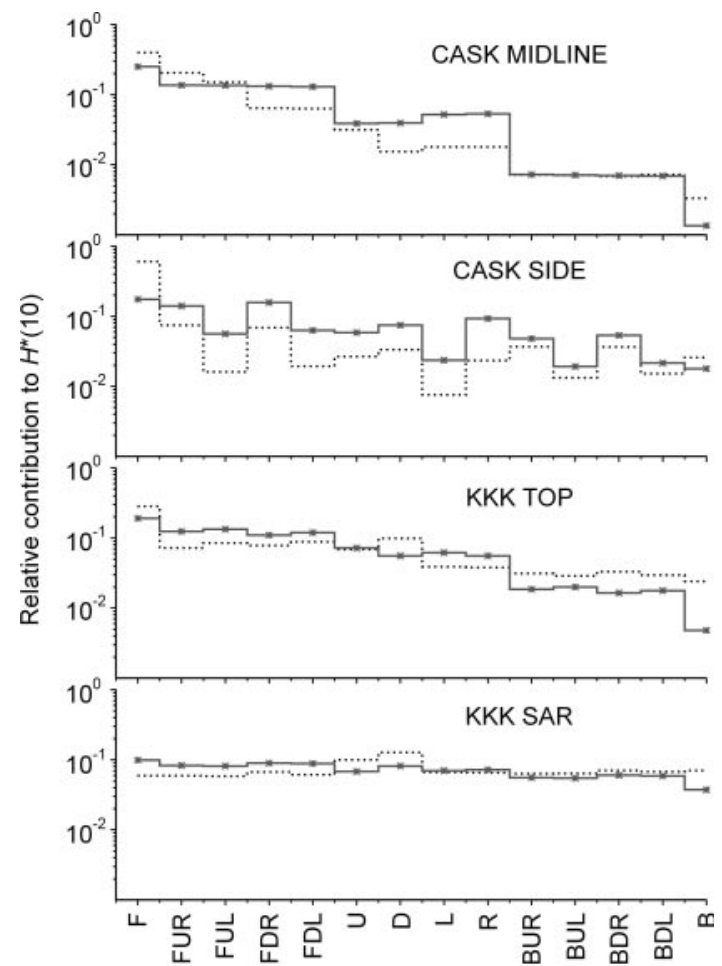

Figure 3. Relative contributions to $H^{*}(10)$ from various directions (see text) using the MIEKE unfolding (dotted lines, neutron/photon unfolding) and the MAXED unfolding (full lines, unfolding with pre-information taken from BSs).

\section{$H_{\mathrm{p}}(\mathbf{1 0})$ and $E$ for different directions of a person's orientation}

Values of $H_{\mathrm{p}}(10)$ and $E$ normalised to $H^{*}(10)$ are shown in Table 2 for six different directions of a person's orientation.

For fields with chiefly directed neutrons $H_{\mathrm{p}}(10)$ values decrease strongly from values close to $H^{*}(10)$ for the 'Front' direction to values about one order of magnitude smaller at the 'Back' direction (see 'cask midline'). They stay almost constant at a value of about one-third of $H^{*}(10)$ in fields with an almost isotropic distribution (see KKK SAR). 
ELECTRONIC NEUTRON DOSEMETERS IN POWER PLANTS

Table 2. Ratio of personal dose equivalent $H_{\mathrm{p}}(10)$ to ambient dose equivalent $H^{*}(10)$ and effective dose $E$ and $E_{\text {new }}$ to ambient dose equivalent $H^{*}(10)$ for a person facing different directions (see text).

\begin{tabular}{|c|c|c|c|c|c|c|}
\hline Position & Front & Up & Down & Left & Right & Back \\
\hline \multicolumn{7}{|l|}{ Cask midline } \\
\hline$H_{\mathrm{p}}(10) / H^{*}(10)$ & $0.78(8)$ & $0.31(4)$ & $0.23(4)$ & $0.23(1)$ & $0.25(2)$ & $0.05(3)$ \\
\hline$E / H^{*}(10)$ & $0.44(5)$ & $0.24(1)$ & $0.24(1)$ & $0.28(1)$ & $0.27(2)$ & $0.31(3)$ \\
\hline$E_{\text {new }} / H^{*}(10)$ & $0.36(5)$ & & & & & \\
\hline \multicolumn{7}{|l|}{ Cask side } \\
\hline$H_{\mathrm{p}}(10) / H^{*}(10)$ & $0.61(14)$ & $0.27(5)$ & $0.31(8)$ & $0.15(5)$ & $0.33(6)$ & $0.18(6)$ \\
\hline$E / H^{*}(10)$ & $0.40(9)$ & $0.27(5)$ & $0.27(4)$ & $0.28(4)$ & $0.28(2)$ & $0.32(6)$ \\
\hline \multirow{2}{*}{\multicolumn{7}{|c|}{$\begin{array}{l}E_{\text {new }} / A(10) \\
\text { KKK TOP }\end{array}$}} \\
\hline & & & & & & \\
\hline$H_{\mathrm{p}}(10) / H^{*}(10)$ & $0.58(5)$ & $0.29(1)$ & $0.29(3)$ & $0.25(2)$ & $0.25(2)$ & $0.10(5)$ \\
\hline$E / H^{*}(10)$ & $0.44(3)$ & $0.32(2)$ & $0.32(2)$ & $0.31(2)$ & $0.31(3)$ & $0.34(3)$ \\
\hline$E_{\text {new }} / H^{*}(10)$ & $0.30(3)$ & & & & & \\
\hline \multicolumn{7}{|l|}{ KKK SAR } \\
\hline$H_{\mathrm{p}}(10) / H^{*}(10)$ & $0.30(2)$ & $0.31(5)$ & $0.34(5)$ & $0.28(2)$ & $0.30(3)$ & $0.28(6)$ \\
\hline$E / H^{*}(10)$ & $0.32(3)$ & $0.35(1)$ & $0.35(1)$ & $0.31(2)$ & $0.31(3)$ & $0.31(2)$ \\
\hline$E_{\text {new }} / H^{*}(10)$ & $0.23(1)$ & & & & & \\
\hline
\end{tabular}

The uncertainties are standard deviations using four different unfoldings (see text).

$E$ values show smaller changes with respect to direction. In fields with chiefly directed neutrons, $H_{\mathrm{p}}(10)$ values overestimate $E$ for 'Front' direction and underestimate $E$ for 'Back' direction (see 'cask midline', factors 1.8 and 6.4). In almost isotropic fields $H_{\mathrm{p}}(10)$ values and $E$ values almost coincide (see KKK SAR).

\section{RESULTS OF PERSONAL DOSEMETERS}

Personal dosemeters (active and passive devices) were irradiated on ISO phantoms in the positions 'cask side', 'cask midline' and 'KKK TOP' for $\sim 2 \mathrm{~h}$ and inside the SAR under nitrogen atmosphere for $38 \mathrm{~h}$. In the latter case, the etched track dosemeters (CR-39 /PADC from PSI and NRPB) were sealed in air in order to avoid track-fading due to oxygen deficiency. The readings of the personal dosemeters attached on the front side are shown in Figure 4. In all cases, dosemeters were also attached on the backside of the phantom. The readings were mostly zero or close to the detection limit of the dosemeters and are not shown in Figure 4 besides those measured at the SAR, where the radiation field was much more isotropic.

The dosemeter readings are shown with respect to values of $H_{\mathrm{p}}(10)$ and $E$ calculated using the $H^{*}(10)$ values given in Table 1 and ratios of $H_{\mathrm{p}}(10) / H^{*}(10)$ and $E / H^{*}(10)$ given in Table 2. The uncertainties given in Tables 1 and 2 are estimated on standard deviation of results of different unfoldings. The absolute uncertainties of $H_{\mathrm{p}}(10)$ and $E$ contain additional uncertainties of the raw data and the instrument calibration itself. A full uncertainty analysis is

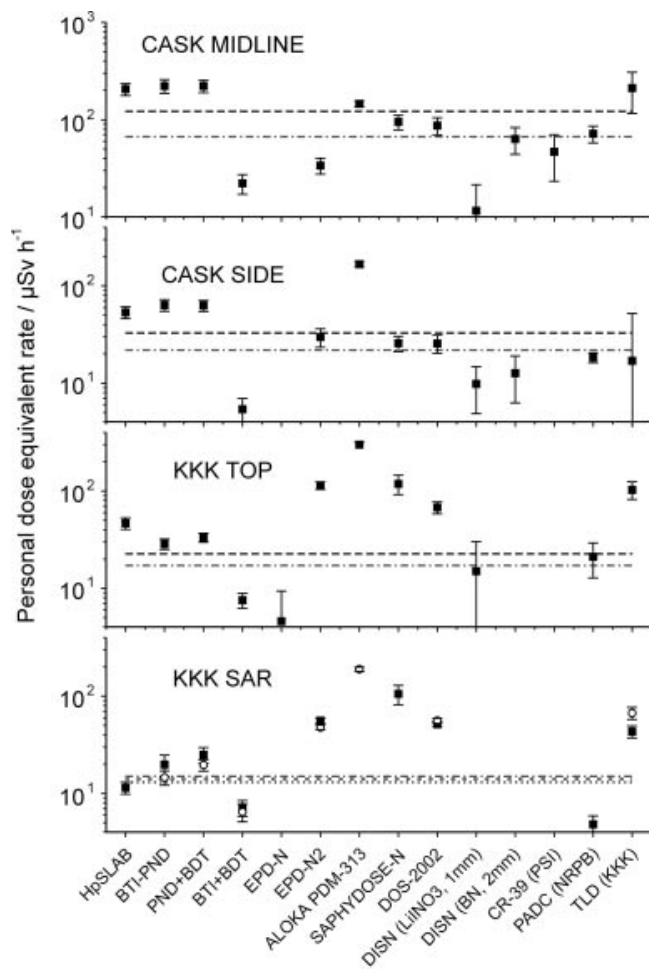

Figure 4. Personal dose equivalent rates $\dot{H}_{\mathrm{p}}(10)$ of dosemeters at Krümmel. The dashed lines indicate estimated values of $\dot{H}_{\mathrm{p}}(10)$, the dashed/dotted lines estimated values of $\dot{E}$. The local device was a TLD albedo dosemeter. The full/open symbols indicate values measured at the front side/back side of the phantom [ $\dot{H}_{\mathrm{p}}(10)$ value dotted for back side]. 
not yet available. A conservative estimate of the uncertainty of $H_{\mathrm{p}}(10)$ and $E$ values is $30 \%$.

The dosemeters based on superheat drop detectors (HpSLAB, BTI-PND and PND-BDT) show in most cases an overresponse (roughly a factor 2) with respect to $\dot{H}_{\mathrm{p}}(10)$ besides the dosemeter devoted to thermal fields (BTI-BDT), which gave lower values.

The electronic dosemeter EPD-N devoted to measuring thermal and intermediate energy neutrons showed very low or zero values in all cases.

The electronic dosemeters (EPD-N2, DOS-2002 and Saphydose-n, all based on one or more silicon detectors) showed reasonable agreement with $\dot{H}_{\mathrm{p}}(10)$ at the cask besides the EPD-N2 that showed too low values at 'cask midline' and values a factor 3-5 times too high inside the reactor.

The electronic dosemeter ALOKA PDM-313 (also based on a silicon detector) showed in all fields with substantial contributions of scattered neutrons much too high values (factor 5-10) compared with $\dot{H}_{\mathrm{p}}(10)$. Only in the case of the position 'cask midline' was the value indicated close to the reference value.

The DISN devices, which are under investigation at PSI, using different wall materials and absorbers, showed in general underreadings compared with $\dot{H}_{\mathrm{p}}(10)$ and high uncertainties owing to a photon subtraction procedure and could not be used in fields with high photon dose (KKK SAR).

The dosemeters based on CR-39/ PADC showed in general values lower than $\dot{H}_{\mathrm{p}}(10)$ or even zero values. In the case of the SAR, the low values may have been caused by track-fading owing to oxygen deficiency.

The 'Local device' used in routine at the Krümmel nuclear power plant is an TLD albedo dosemeter and was used with calibration factors as foreseen for the different application areas (N1 inside the power plant and N2 at the cask). At the cask, the values agreed within the uncertainties with $\dot{H}_{\mathrm{p}}(10)$, while inside the power plant, $\dot{H}_{\mathrm{p}}(10)$ is overestimated by a factor of $3-4$.

With respect to $\dot{E}$, the overresponse observed with electronic dosemeters inside the power plant stays at almost the same level, since $\dot{H}_{\mathrm{p}}(10)$ and $\dot{E}$ do not deviate strongly. At the cask, the dosemeter readings become more conservative with respect to $\dot{E}$.

This conservative behaviour of personal dosemeter readings with respect to $\dot{E}$ will still increase if new radiation weighing factors $w_{\mathrm{R}}$ as recently proposed by members of the ICRP ${ }^{(8)}$ are used. The resulting values of $E_{\text {new }}$ are given in Table 2 in the last column. They are $\sim 20 \%$ (cask) to $40 \%$ (reactor) lower than the old values.

\section{CONCLUSIONS}

Depending on the radiation field, $H_{\mathrm{p}}(10)$ is only between 30 and $80 \%$ of $H^{*}(10)$. Most of the electronic dosemeters show conservative (too high) readings in the fields investigated at the Krümmel reactor.

\section{ACKNOWLEDGEMENT}

This research is partly funded by the European Commission under the auspices of the Euratom 5th Framework Programme: Nuclear Energy, 1998-2002, Contract No. FIKR-CT-2001-00175.

\section{REFERENCES}

1. Bolognese-Milsztajn, T. et al. Individual neutron monitoring in workplaces with mixed neutron/photon radiation. Radiat. Prot. Dosim. 110, 753-758 (2004).

2. Luszik-Bhadra, M. et al. EVIDOS: optimisation of individual monitoring in mixed neutron/photon fields at workplaces of the nuclear fuel cycle. In: 4th ISOE European workshop on occupational exposure management at NPPs, Lyon, France, 24-26 March 2004. Available on http://isoe.cepn.asso.fr/. Copyright CEPN 1998-2005.

3. Vanhavere, F. et al. Evaluation of individual monitoring in mixed neutron/photon fields: mid-term results from the EVIDOS project. Radiat. Prot. Dosim. 120(1-4), 263-267 (2006).

4. Luszik-Bhadra, M., Reginatto, M. and Lacoste, V. Measurement of energy and direction distribution of neutron and photon fluences in workplace fields. Radiat. Prot. Dosim. 110, 237-241 (2004).

5. Reginatto, M., Luszik-Bhadra, M. and d'Errico, F. An unfolding method for directional spectrometers. Radiat. Prot. Dosim. 110, 539-543 (2004).

6. International Commission on Radiological Protection. Conversion coefficients for use in radiological protection against external radiation. ICRP Publication 74. Ann ICRP 26 (3-4) (Oxford: Pergamon Press) (1996).

7. d'Errico, F., Giusti, V. and Siebert, B. R. L. A new neutron monitor and extended conversion coefficients for $H p$ (10). Presented at the IM2005 European Workshop on individual monitoring of ionising radiation, Vienna, April 11-15 (2005). Radiat. Prot. Dosim. (to be published).

8. Dietze, G. and Harder, D. Proposal for a modified radiation weighting factor for neutrons. In: Proceedings of the 11th International Congress of the International Radiation Protection Association, 23-28 May 2004, Madrid, Spain. Published on CD. Produced in Spain by SENDA. ISBN: 84-87078-05-2. 